\title{
Estudo comparativo da análise hierárquica com multiobjetivo para seleção de projetos públicos de investimento em infra-estrutura de transporte
}

\author{
Cristianne da Silva Macêdo; Joel Castro do Nascimento²; Nelson Kuwahara ${ }^{3}$
}

\begin{abstract}
Resumo: Este artigo apresenta um estudo comparativo do Método de Análise Hierárquica, aplicado ao estado do Amazonas, associando com Método de Programação Matemática Multiobjetivo o qual foi aplicado para o caso de Bogotá. Na pesquisa bibliográfica, realizou-se levantamento e análise de métodos que foram aplicados em problemas de investimentos públicos. Souza (1994), mostrou que erros de planejamentos resultaram em fracassos nos esperados benefícios esperados para a rodovia Transamazônica. Este registro exemplifica que equivocados planejamentos em investimentos públicos, associado com o não envolvimento dos atores locais, resultam em prejuízos para a sociedade.
\end{abstract}

Abstract: This paper show Hierarchical Analysis Method applied in the state of Amazonas, mixed with Programming Mathematical Multiobjective Method, that was modeled to case of Bogota. The study developed analysed some public investments, considering indicated methods. Souza (1994), by way of example, in this question, showed mentioned mistake of project road in Amazon, called Transamazonica, who linked only two brazilian regions without benefic effects. This example proved that wrong planning in public investments, associeted with not participating of stakeholders, happening in not benefics to society.

\section{INTRODUÇÃO}

É possível enxergar o papel do transporte na criação de alto nível de atividade econômica a partir da comparação das economias de uma nação desenvolvida e de outra em desenvolvimento. Deve-se salientar que o aumento da competitividade de mercado, a garantia da economia de escala na produção e redução dos preços das mercadorias é resultado de um melhor sistema de transporte (Ballou, 2008). Nota-se que a infraestrutura de transporte é um diferencial competitivo para as empresas e governo de um determinado país.

Porém, no Brasil a inadequada e insuficiente infraestrutura colabora para uma baixa participação nas exportações, sendo da ordem de $1 \%$, tornando o país limitado para o crescimento e expansão de sua economia, conforme Erhart e Palmeira (2006). Especificamente no Amazonas, cercado por seus extensos rios, a sua realidade não é diferente dos demais estados do país, pois as suas características peculiares de condições climáticas e ambientais contribuem para a adversidade na sua acessibilidade, tendo em vista que os portos da Amazônia, exceto os das capitais e alguns municípios, são inapropriados para a movimentação

\footnotetext{
${ }^{1}$ Cristianne da Silva Macêdo, Laboratório TRANSPORTAR, Universidade Federal do Amazonas, Manaus, AM, Brasil. (e-mail: cristianne_silvamacedo@yahoo.com.br).

${ }^{2}$ Joel Castro do Nascimento, Laboratório TRANSPORTAR, Universidade Federal do Amazonas, Manaus, AM, Brasil. (e-mail: jcastro_ep@yahoo.com.br).

${ }^{3}$ Nelson Kuwahara, Laboratório TRANSPORTAR, Universidade Federal do Amazonas, Manaus, AM, Brasil. (e-mail: nelsonk@ufam.edu.br).

Manuscrito recebido em 12/10/2009 e aprovado para publicação em 4/5/2010. Este artigo é parte de TRANSPORTES, volume XVIII, número 2, junho de 2010. ISSN: 2237-1346 (online).
}

de cargas e pessoas (Kuwahara, 2006).

Os problemas expostos anteriormente ocorrem devido à superficialidade na tomada de decisão de investimentos em projetos públicos, pois o governo não dispõe de subsídios para tomar decisão de forma mais eficaz e eficiente. Desta forma, o mesmo necessita de ferramentas para auxiliar na seleção de projetos públicos com o intuito de obter melhorias substanciais para a sociedade.

Sendo assim, este artigo tem por objetivo realizar uma abordagem teórica das aplicações do Método de Programação Matemática Multiobjetivo - MPMM e Método de Análise de Decisão (MAD) para seleção de projetos de investimento em infra-estrutura de transporte a fim de analisar a eficácia da utilização conjunta dessas ferramentas para a tomada de decisão.

\section{PLANEJAMENTO DOS INVESTIMENTOS EM PROJETOS PÚBLICOS DE INFRA- ESTRUTURA DE TRANSPORTE}

Segundo Closs \& Bowersox (2007), a infra-estrutura de transporte deve fornecer direito ao acesso, tanto de veículos como de unidades organizacionais de transportes a fim de munir de serviços para uso próprio ou para terceiros, mediante a cobrança de taxa de serviço, trazendo variados benefícios para a sociedade, como por exemplo, à disponibilidade de bens, à ampliação dos mercados, à concorrência, aos custos dos produtos, entre outros (Caixeta Filho \& Martins, 2001).

Em vários países do mundo, inclusive no Brasil, a oferta de infra-estrutura, foi, e ainda é importante para o desenvolvimento da região. Segundo Kuwahara et al. (2008), o Brasil é usuário do Método Multicriterial 
(MCA) para determinar a viabilidade dos projetos de investimentos em transportes, mas não partilha o poder de tomada de decisão de alocação dos recursos, e também essas decisões são realizadas sem base na demanda e interesse do stakeholders. Informam, ainda, os autores que desde 1988 os investimentos públicos no Brasil são planejados pelo Governo Federal através dos Planos Plurianuais (PPA's) em quadriênios formulados pela Lei incentivadora do Executivo.

Os investimentos previstos nos PPA's são responsáveis em garantir a efetivação da melhoria das infraestruturas proporcionando ao país sua transformação, além disso, para o apoio ao desenvolvimento econômico o governo lança programas ou ações para tal fim (Kuwahara, 2008). Porém, as Leis de Diretrizes Orçamentárias (LDO), os orçamentos anuais, planos e programas nacionais, regionais e setoriais devem estar de acordo com o que está regido no Plano Plurianual daquele período de governo (Garcia, 2000). Contudo, tais planos não possuem metodologia adequada para estabelecer os investimentos por Estado colaborando para as desigualdades entres os mesmos.

No Estado do Amazonas, por exemplo, as áreas de Planejamento de Transporte e Logística têm sido as principais causas que interferem diretamente no desenvolvimento econômico da Região, especificamente em relação ao Pólo Industrial de Manaus - PIM e o interior do estado (Portugal, 2006), pois a infra-estrutura disponibilizada para escoar a produção torna o custo de transporte mais elevado acarretando na baixa competitividade no mercado.

No próprio PPA 2008-2011 do Estado do Amazonas, a Secretaria de Estado de Planejamento e Desenvolvimento Econômico - SEPLAN (2007) afirma que o modelo de transporte intermodal tem sido o mais utilizado e que o mesmo precisa ter sua estrutura portuária no interior e na capital mais fortalecida a fim de obter melhor fluxo comercial entre produtores e centros consumidores tanto a nível regional como nacional e internacional.

\section{MODELOS E FERRAMENTAS APLICADAS NO PLANEJAMENTO}

Neste item serão abordados os conceitos das principais ferramentas e métodos utilizados para a tomada de decisão, dentre elas destacou-se o Apoio Multicritério à Decisão. Esta busca o estabelecimento de relações de preferências entre as alternativas postas em análise pelos tomadores de decisões as quais possuem influências de diversos critérios (Almeida, 2003).

As alternativas indicadas são colocadas perante as partes interessadas, as diferentes possibilidades e respectivos critérios, viabilizam escolha da melhor decisão, sendo que os stakeholders podem interferir no processo decisório.

Segundo Gomes et al. (2002, p. 63), esta é uma das características típicas desses problemas "a solução do problema depende de um conjunto de pessoas, cada uma das quais tem seu próprio ponto vista, muitas vezes conflitante com os demais".

De acordo com Malczewski apud Gomes et al. (2002), tal método envolvem os seguintes componentes:
a. Objetivo;
b. decisor (es);
c. conjunto de critérios de decisão;
d. conjunto de alternativas;
e. conseqüências das decisões.

Dessa forma, o método Multicritério tem como principal objetivo, tomar as decisões baseadas na descrição dos critérios, em que cada um tem suas alternativas com seus respectivos pesos. Esses pesos definirão qual a melhor decisão a ser tomada. Quando as decisões são tomadas em grupos os pesos ou preferências individuais são combinados para formar uma decisão conjunta do grupo (Gomes et al., 2002).

O Método de Análise Multicritério subdivide-se em Método de Programação Matemática Multiobjetivo MPMM e Método de Análise de Decisão (MAD) (Caixeta-Filho e Martins, 2001). Mas para a melhor compreensão de tais métodos faz-se necessário, primeiramente, abordar o processo de tomada de decisão.

\subsection{O Processo de Tomada de Decisão}

Gomes et al. (2002) dizem que é necessário a existência de um conjunto de alternativas viáveis para que ocorra o processo de decisão, sendo que em cada alternativa há fatores positivos e negativos, podendo ser, por exemplo, maximização do lucro ou minimização dos custos. No caso de investimentos em projetos públicos, esta tomada de decisão torna-se mais complexa, devido os resultados abrangerem de forma satisfatória os seguintes aspectos: econômicos, sociais, políticos e ambientais.

Essa complexidade se dá devido o atendimento a múltiplos objetivos, sendo que tais decisões complexas tornam-se mais difíceis e não se podem identificar corretamente os seus impactos (Gomes et al., 2002). No caso dos empreendimentos públicos, Medaglia (2008) afirma que são três os problemas comuns enfrentados por tais empresas, o primeiro é a questão do orçamento disponível para os projetos de investimento os quais são insuficientes para o início de todos os projetos durante o primeiro ano e o segundo está nas limitações técnicas, como por exemplo, as datas de início e as de término, e, por fim, as relações de precedências entre projetos específicos.

Seja qual for o tipo de investimento, o importante 
para o decisor, pessoa que influencia no processo de decisão, é compreender que cada problema possui sua natureza e entendê-la é peça fundamental para melhor solução do mesmo (Gomes et al., 2002).

\subsection{Método de Análise de Decisão - MAD}

No Método de Análise de Decisão - MAD as restrições são implícitas e as soluções fazem parte do conjunto discreto (KUWAHARA, 2008), ou seja, as restrições são determinadas de acordo com o grau de importância para os stakeholders e suas soluções são conhecidas pelos mesmos sendo apenas determinada a mais satisfatória.

As decisões podem ser tomadas baseadas em alguns métodos que Caixeta-Filho e Martins (2001) destacam os seguintes:

- ELECTRE (Elimination et Choix Traduissant la Realité);

- MAC (Método de Análise de Concordância);

- PROMETHEE (Preference Ranking Organization Method);

- TOPSIS ((Technique Order Preference by Similarity to Ideal Solution);

- TODIM (Tomada de Decisão Interativa e Multicritério);

- MAH (Método de Análise Hierárquica).

Porém, neste trabalho será dada ênfase ao Método de Análise Hierárquico devido o mesmo "possuir uma técnica que reduz o risco de manipulação no processo decisório e possibilitar a obtenção de julgamentos consistentes, por meio da revisão orientada", conforme Jardim (1999, p. 31).

O MAH foi desenvolvido na década de 70 por Thomas L. Saaty a partir da necessidade de obter uma solução para o problema específico de planejamento de contingência e posteriormente sua aplicação se deu no planejamento de alternativas futuras para um país em desenvolvimento (Saaty, 1991).

De acordo com Jardim (1999), este método classifica as alternativas por meio da comparação aos pares, utilizando para isso matrizes quadradas baseadas nas propriedades algébricas matriciais, tais matrizes são recíprocas, positivas e não devem conter zero.

\subsection{Método de Programação Matemática Multiobjetivo - MPMM}

Segundo Jardim (1999), o modelo Multiobjetivo seleciona a solução de melhor compromisso com os objetivos apresentados, onde se busca a otimização do conjunto das funções-objetivo com suas respectivas restrições, através dos critérios e alternativas na solução do problema. Esse modelo utiliza ferramentas da Pesquisa Operacional, tais como programação linear e não-linear, para assim chegar a uma solução mais adequada para todos os objetivos, sempre levando em conta a incerteza e a subjetividade que estão inerentes ao processo de tomada de decisão.

As soluções desse tipo de modelo dividem-se em: dominada e não-dominada, conforme a aplicabilidade do Princípio de Otimalidade de Pareto (JARDIM, 1999). Porto e Azevedo apud Jardim (1999, p.5) afirmam que uma solução dominada é a região viável das soluções e não-dominada "é aquela em que a melhoria de uma função-objetivo só pode ser conseguida às custas da degradação da outra".

Tal modelo pode ser chamado também de um processo de otimização por vetor, como a estrutura que se segue (Cohon e Marks apud Jardim, 1999, p.6):

$$
\operatorname{Max} Z(x)=\left\lfloor Z_{1}(x), Z_{1}(x), \ldots, Z_{p}(x)\right\rfloor,
$$

sujeito a:

$$
\begin{aligned}
& g_{i}(x) \leq 0 \quad i=1,2,3, \ldots, m \\
& x_{j} \geq 0 \quad j=1,2,3, \ldots, n
\end{aligned}
$$

em que,

$Z(x)$ : função-Objetivo p-dimensional;

$p$ : número de objetivos;

$g_{i}(x)$ : função-restrição m-dimensional;

$x_{j}(x)$ : vetor-dimensional das variáveis de decisão.

Para o conjunto $X$ das soluções viáveis no espaço das variáveis de decisão, tem-se:

$$
\operatorname{Max} \sum_{l=1}^{3} \lambda l \cdot \sum_{i \in P} \sum_{i=t_{i}^{-}}^{\min \left\{t_{i t}^{+}, T-u_{i}+1\right\}} L_{i t}^{l},
$$

Uma solução não-dominada $x$ é uma solução viável do conjunto $X$, não havendo outra solução viável $x^{\prime} €$ $X$, em que ocorram:

$$
Z_{r}\left(x^{\prime}\right)>Z_{r}(x) \text { para algum } r=1,2, \ldots, p
$$

e $Z k\left(x^{\prime}\right) \geq Z k(x)$ para todo $k$ diferente de $r$.

$\mathrm{O}$ conjunto $X^{*}$ das soluções não-dominadas é representado por:

$$
X^{*}=\{x / x \in X \text { e } x \text { conforme definido acima }\}
$$

\section{EXPERIÊNCIAS DE APLICAÇÕES}

Sabe-se que são muitas as aplicações dos métodos de análise de decisão e de programação matemática multiobjetivo no âmbito do setor privado, porém no público ainda há certa resistência na sua utilização. Desta forma, neste item, serão relatados os modelos propostos por dois autores e suas aplicações em tal setor.

\subsection{Experiência da aplicação do Método de Análise de Decisão - ênfase ao MAH}

O método de Análise de Decisão foi utilizado por Kuwahara por meio do Método de Análise Hierárquica, com o objetivo de propor um modelo de planejamento de investimentos em infra-estrutura de transportes no estado do Amazonas. A primeira etapa do seu trabalho deu-se pela consulta as partes interessadas com o assunto abordado, para assim obter a estru- 
tura de hierarquização e a montagem das matrizes a fim de analisar aos pares as alternativas de transportes a partir dos critérios, ambientais, sociais e econômicos levantados.

Os critérios específicos foram: Expansão de Malha Viária Urbana - EMVU; Diversificação de Atividade Produtiva - DAP; Impactos Ambientais Resultantes do Uso da Obra - IARUO; Geração de Empregos Relacionada com as Atividades Produtivas Dependentes - GEAPD; Relação Custo/Benefício - RCB; Acessibilidade Intermunicipal, Interestadual e Internacional AIII.

As alternativas analisadas em tal modelo de infraestrutura foram: Ampliação Setor de Cargas do Aeroporto Eduardo Gomes - ASCEG; Construção do Terminal Bi-Modal da CEASA de Manaus - CTBCM; Malha viária de Manaus - MVM; Ponte sobre o Rio Negro para interligação do município de Manaus com o município de Iranduba - PMI; Portos nos Municípios do Amazonas - PMA; Reconstrução da Rodovia BR 319, interligando Manaus/AM com Porto Velho/RO - BR-319.

Foram realizadas três análises, a primeira em relação ao aspecto ambiental, a segunda o social e a terceira, o aspecto econômico. A primeira deu-se através das seguintes percentagens de importância dos critérios, estabelecidas pelos stakeholders, na ordem decrescente: (1) IARUO: 33,6\%, (2) RCB: 31,6\%, (3) GEAPD: $13,7 \%$, (4) DAP: 10,3\%, (5) AIII: 8,5\% e (6) EMVU: $2,2 \%$. Ao simular os dados no Expert - Choi$c e$, software de apoio a tomada de decisão, os resultados obtidos na ordem decrescente, foram: (1) Portos nos Municípios do Amazonas - PMA: 26,4\%, (2) Construção do Terminal Bi-Modal da CEASA de Manaus - CTBCM: 23,5\%, (3) Ampliação Setor de Cargas do Aeroporto Eduardo Gomes - ASCEG: 20,7\%, (4) Malha viária de Manaus - MVM: 18,6\%, (5) Ponte sobre o Rio Negro para interligação do município de Manaus com o município de Iranduba - PMI: 6,4\% e (6) Reconstrução da Rodovia BR 319, interligando Manaus (AM) com Porto Velho (RO) - BR319: 4,4\%.

$\mathrm{Na}$ segunda análise os pesos de AIII e GEAPD foram de 38,9\% e 37,2\%, respectivamente, obtendo-se os seguintes resultados, de acordo com o grau de importância para o empreendimento: (1) PMA: 28,3\%, (2) CTBCM: 24,4\%, (3) ASCEG: 23,3\%, (4) BR-319: 9,3\%, (5) PMI: 8,3\% e (6) MVM: 6,3\%. Já na terceira análise os pesos de RCB e GEAPD obtiveram os valores de $56,5 \%$ e $16 \%$, nesta ordem, alcançando os resultados a seguir: (1) PMA: 26,9\%, (2) CTBCM: 23,3\%, (3) MVM: 18,8\%, (4) ASCEG: 18,5\%, (5) PMI: $8,6 \%$ e (6) BR-319: 3,9\%.

Tais análises permitiram que o autor chegasse à conclusão que o modelo possibilita a transparência, legitimidade e eficiência no planejamento de investi- mentos em infra-estrutura de transporte para o estado do Amazonas e que ao comparar as alternativas apontadas pelo poder público como o mais importante há controvérsias com os resultados simulados.

\subsection{Experiência da aplicação do Modelo Multiobjetivo}

A aplicação do modelo multiobjetivo foi realizado por Medaglia et al. (2008) em que seu objetivo foi expandir o conjunto de ferramentas de tomada de decisão à disposição dos órgãos públicos para a seleção e programação de projetos por meio de um programa multiobjetivo linear inteira mista, conforme mostra a equação 3.

Conforme Medaglia et al. (2008), o modelo apresentado inclui a formulação de um conjunto de projetos possíveis a serem realizados, $P$; o mínimo e o máximo número de projetos a serem incluídos no portifólio, $K_{L}$ e $K_{U}$, respectivamente, e um conjunto de precedência das relações entre os projetos, $A$. Ou seja, se o projeto $i \in P$ antecede o projeto $j \in P$, então $(i, j) \epsilon$ $A$, cuja precedência diferencial é, por sua vez, representada por $g_{i j} \in Z_{+}{ }^{l}$. Se $g_{i j}=0$, o último investimento do período do projeto $i$ deve ser antes do início do investimento ao longo do ciclo do projeto $j$; se $g_{i j}<0$, uma sobreposição de até $g_{i j}$ períodos de investimento serão permitidos, e, se $g_{i j}>0$, o último período de investimento do projeto $i$ deve ser de pelo menos $g_{i j}$ períodos de distância a partir do primeiro período de investimento do projeto $j$.

A vida do projeto $i$ é definido por $v_{i}$, e o tempo de investimento (ou seja, o número de períodos até o último período de investimento) por $u_{i} . T$ representa o horizonte de planejamento de decisões de investimento $(t \epsilon\{0, \ldots, T\})$, enquanto $\lambda_{1}, \lambda_{2}$ e $\lambda_{3}$ são os pesos dado a priori pelo decisor aos respectivos fatores econômicos, financeiros e sociais, respectivamente $(0 \leq$ $\left.\lambda_{1} \leq 1 ; \sum_{i=1}^{3} \lambda_{1}=1\right)$.

A normalização dos índices econômicos, financeiros, e social são, respectivamente, $I_{i t}^{1}, I_{i t}^{2}$ e $I_{i t}^{3}$ se projeto $i \in P$ começa no ano $t\left(t \in\left\{t_{i}^{-}, \ldots, \min \left\{t_{i}^{+}, T-u_{i}\right.\right.\right.$ $+1\}\})$. Finalmente, $C_{i k}$ e $B_{i k}$ são os custos de investimento e de benefícios no período k para projeto $i$ (k $\epsilon$ $\left.\left\{0, \ldots, v_{i}-1\right\}\right) ; t_{i}^{-}$e $t_{i}^{+}$são as primeiras e as últimas datas de início respectivas para o projeto $i$ ( $i \in P$ ); e $r_{T}^{0}$ é o orçamento disponível para o ano $t(t \epsilon\{0, \ldots, T\})$. Sem perda de generalidade, assumimos que $r_{T}^{0}=0$ (Medaglia et al., 2008).

O modelo, proposto por Medaglia et al. (2008), identifica os projetos a serem selecionados e, o início da data de cada um. A decisão variável binária, $y_{i t}$, tem um valor de 1 caso o projeto $i \in P$ se iniciar no ano $\left(t \in\left\{t_{i}^{-}, \ldots, \min \left\{t_{i}^{+}, T-u_{i}+1\right\}\right\}\right)$ e um valor de 
0 , de outra forma. O modelo exige um conjunto auxiliar de variáveis binárias de decisão, $x_{i k t}$, que têm o valor de 1 , caso o período ( $\left.\mathrm{k} \epsilon\left\{0, \ldots, v_{i}-1\right\}\right)$ para o projeto $i$ \& $P$ seja atribuído ao ano t, no horizonte de planejamento $\left(t \in\left\{t_{i}^{-}, \ldots, \min \left\{t_{i}^{+}, T-u_{i}+1\right\}\right\}\right)$, e um valor de 0 , de outra forma. Permitindo $r_{t}(\geq 0)$ ser os recursos disponíveis no ano $(t \in\{0, \ldots, T+1\})$. Assumimos, sem perda de generalidade, que $r_{0}=0$.

$$
\operatorname{Max} \sum_{l=1}^{3} \lambda l \cdot \sum_{i \in P} \sum_{i=t_{i}^{-}}^{\min \left\{t^{+}, T-u_{i}+1\right\}} L_{i t}^{l} \cdot y_{i t},
$$

Sujeito a

$$
\begin{gathered}
\sum_{t=t_{i}^{-}}^{\min \left\{t t_{i}^{+}, T-u_{i}+1\right\}} \leq 1, i \in P, \\
K_{L} \leq \sum_{i \in P} \sum_{t=t_{i}^{-}}^{\min \left\{t_{i}^{+}, T-u_{i}+1\right\}} y_{i t} \leq K_{U}, \\
y_{i t}=x_{i}, k, t+k, i \in P, k \in\left\{0, \ldots, v_{i}-1\right\}, \\
t \in\left\{t_{i}^{-}, \ldots, \min \left\{t_{i}^{+}, T-u_{i}+1\right\}\right\} \\
y_{i t} \leq \sum_{t^{\prime}=t_{i}^{-}}^{t-u_{i}-g_{i j}} y_{i t},(i, j) \in A, t \in\left\{t_{i}^{-}, \ldots, t_{i}^{+}\right\}, \\
r_{t+1}=r_{t}+r_{t}^{0}+\sum_{i \in P} \sum_{k=0, \ldots, v_{i}}\left(b_{i k}-c_{i k}\right) \cdot x_{i k t}, t \in\{0, \ldots, T\}, \\
r_{t} \geq 0, t \in\{0, \ldots, T+1\}, \\
y_{i k t} \in\{0,1\}, i \in P, t \in\left\{t_{i}^{-}, \ldots, \min \left\{t_{i}^{+}, T-u_{i}+1\right\}\right\}, \\
x_{i k t} \in\{0,1\}, i \in P, k \in\left\{0, \ldots, v_{i}-1\right\}, t \in\left\{t_{i}^{-}, \ldots, t_{i}^{+}+v_{i}-1\right\} .
\end{gathered}
$$

A equação (3) mostra o objetivo do modelo que visa a soma ponderada dos valores econômicos, financeiros, e social do portifólio normalizados. As restrições em (4) permitem que o modelo escolha, no máximo, uma data de início de cada projeto. As restrições vinculadas na (5) permite que o número de projetos selecionados esteja entre um limite inferior e um limite superior. Se estes dois limites são iguais, o número de projetos a serem selecionados é fixo. O grupo de restrições em (6) articula decisão de variáveis $y$ com variáveis auxiliares $x$. Se um determinado projeto começa em uma determinada data, a variável correspondente $y$ assume o valor de 1 . Assim, os períodos de projeto são atribuídos a determinadas datas no horizonte de planejamento através da ativação das variáveis correspondentes $x$ (Medaglia et al., 2008).

As condições de precedência são contabilizadas em (7) e as restrições em (8) são responsáveis pela reuni- ão do orçamento exógenos incorporando o reinvestimento dos recursos endógenos gerados por outros projetos. Finalmente, o grupo de limitações em (9), estabelece as condições de não-negatividade para os recursos disponíveis no final de ano $t$, enquanto que aqueles em (10) e (11) força as variáveis $x$ e $y$ para ser binárias (Medaglia et al., 2008).

$\mathrm{O}$ índice social foi medido por Medaglia et al. (2008) diretamente pelo número de pessoas abaixo da linha da pobreza as quais o projeto irá beneficiar. Considerou-se que tal empresa possuía $10(\mathrm{dez})$ projetos com um horizonte temporal de 8 (oito) anos e levou-se em conta que 5 (cinco) desses projetos, $\mathrm{p}_{3}, \mathrm{p}_{5}$, $\mathrm{p}_{6}$ e $\mathrm{p}_{7}$, não possuem flexibilidade quanto as suas datas de início.

Os autores analisam a sensibilidade quanto aos pesos dos objetivos, a sensibilidade às limitações técnicas e sensibilidade a alterações orçamentais. Para análise da sensibilidade quanto aos pesos dos objetivos foi avaliado três cenários, analisando os pesos dependendo do que o tomador de decisão deseja, caso almeje beneficiar maior quantidade de pessoas abaixo da linha da pobreza o quanto ele terá que sacrificar na eficiência econômica do seu projeto.

A importância do modelo supracitado é evidenciada, segundo os autores, por permitir a seleção ótima e ordenação de um cenário anterior, quantitativamente mede o custo de oportunidade de decisões políticas e facilita o processo de planejamento das empresas públicas que investem os recursos públicos voltados para o bem-estar social.

\section{ESTUDO COMPARATIVO DO MÉTODO DE ANÁLISE DE DECISÃO (MAD) COM O MÉTODO DE PROGRAMAÇÃO MATEMÁTICA MULTIOBJETIVO - MPMM}

O Método de Análise de Decisão auxilia de forma qualitativa e, de certa forma, quantitativa o tomador de decisão a obter a solução satisfatória dentre as alternativas e critérios definidos pelas partes interessadas. Outras características deste método são, conforme Caixeta-Filho (2001), apresentar restrições implícitas, pois de acordo com os objetivos do sistema inicialmente definido se uma alternativa não estiver compatível com os mesmos a alternativa será descartada, ter as alternativas apresentadas de formas explícitas, devido serem definidas inicialmente, e suas soluções serem constituídas por um conjunto discreto, pois os números de alternativas são finitos.

Ao passo que o de Programação Matemática Multiobjetivo auxilia o tomador de decisão a obter de forma quantitativa a solução de melhor compromisso com seus objetivos, uma vez que segundo Caixeta-Filho (2001) o MPMM apresenta restrições matematicamente explícitas, porque se tratando de programação linear 
é necessário definir primeiramente a função objetivo juntamente com suas restrições para que assim obtenha a solução ótima do problema, com suas alternativas de soluções implícitas, pois só é possível obter as alternativas após a simulação do modelo definido, e a constituição por meio de suas soluções de um conjunto contínuo, pois apresentam infinitos números de alternativas.

Pode-se verificar pelos parágrafos anteriores que a principal diferença entre os métodos analisados está no fato de que o MAD possibilita a intervenção de agentes externos no sistema decisório, por isso as alternativas são explícitas, ao contrário do MPMM que a solução para o problema é dada mediante a formulação das funções-objetivos e de suas respectivas restrições sem a participação direta das partes interessadas.

$\mathrm{Na}$ aplicação do MAD ênfase ao MAH no item 4.1 a característica notória é a participação dos stakeholders, sendo de suma importância para o a definição dos objetivos e levantamento dos critérios. Com isso, as alternativas estão explícitas, onde são finitas, no caso seis, com isso a solução satisfatória pertencem a um conjunto discreto, modificando-se de acordo com o grau de importância dos critérios para as partes interessadas. Neste modelo fica evidente que não é imprescindível para o MAD ter amplo ferramental matemático, visto que o software já possui de forma implícita o algoritmo para realizar a simulação. Sendo necessário somente imputar os dados, objetivo, critérios e alternativas, nas interfaces do programa utilizado, e o mesmo se encarrega de hierarquizar as alternativas.

Já na aplicação multiobjetiva citada no item 4.2 é possível identificar as características do MPMM, pois foi primeiramente elaborado o modelo para a sua programação com suas restrições explícitas e alternativas implícitas, pois conforme se variavam os pesos dos objetivos as mesmas eram sendo expostas ao avaliador do processo decisório, sendo que os autores utilizaram a programação multiobjetiva linear inteira mista para implementar o modelo e obter a solução ótima a qual pertence a um conjunto contínuo. O tomador de decisão ao utilizar tal simulação pôde avaliar os impactos de suas decisões antes das mesmas serem implementadas, principalmente se tratando de investimentos públicos.

\section{CONSIDERAÇÕES FINAIS}

Este artigo procurou comparar dois métodos multicritério, o MAH e o MPMM, a fim de se verificar suas reais aplicabilidades para os investimentos do setor público na área de infra-estrutura de transportes.

Pôde-se verificar que tanto o MAH como o MPMM são possíveis de serem utilizados pelos órgãos respon- sáveis a fim de tomar as decisões da aplicação dos recursos públicos disponibilizados dentro de um determinado período, que no caso do Brasil tem-se estabelecido pelo Plano Plurianual, como explicado no desenvolvimento deste trabalho.

As diferenças dos dois métodos que, está no fato de o MAH possibilitar participações externas e o MPMM não possuir tal flexibilidade, podem ser complementadas ao utilizar os métodos em conjunto a fim de fornecer maior subsídio para as pessoas que estarão frente às alternativas de investimento, principalmente se tratando de problemas de tomada de decisão na área de transportes, os quais não podem ser baseados somente com modelos puramente matemáticos, mas tornando necessário a participação das partes interessadas.

Portanto, é de suma importância em um cenário público, onde há variáveis discretas e contínuas, trabalhar em conjunto com os métodos multicritérios, MAH e MPMM, para que assim haja a eficácia e eficiência na tomada de decisão, principalmente no Brasil por se tratar de um país em desenvolvimento, cuja infra-estrutura de transporte tem um grande impacto no seu crescimento econômico.

\section{AGRADECIMENTOS}

Os autores agradecem o Conselho Nacional de Desenvolvimento Científico e Tecnológico - $\mathrm{CNPq}$, entidade governamental brasileira promotora do desenvolvimento científico e tecnológico, pelo apoio material e financeiro no desenvolvimento do trabalho em questão e ao Centro de Referência em Logística e Planejamento de Transporte de Cargas da Amazônia CRLPTC, por dar subsídios a este trabalho.

\section{REFERÊNCIAS BIBLIOGRÁFICAS}

Almeida, A. T. (2003) A Utilização de Métodos Multicritério de Apoio a Decisão. In: Aplicações com Métodos Multicritérios de Apoio à Decisão. Editora Universitária UFPE, Recife, p.1-19.

Ballou, R. H. (2008) Logística Empresarial: Transportes, Administração de Materiais e Distribuição Física. Editora Atlas, São Paulo.

Bowersox, D. J. e Closs, D. J. (2007) Logística Empresarial: O processo de Integração da Cadeia de Suprimento. $\left(1^{\mathrm{a}} \mathrm{Ed}\right.$. $)-5^{\mathrm{a}}$ reimpr. Editora Atlas, São Paulo, SP.

Caixeta-Filho, J.V. e Martins, R. S. (2001) Logística, Transporte de Carga, Tomada de Decisão em Investimentos. ( $1^{\mathrm{a}} \mathrm{Ed}$.). Editora Atlas, São Paulo, SP.

Erhart, S. e Palmeira, E. M. (2006) Análise do setor de transportes. Observatório de la Economía Latinoamericana: Revista acadêmica de economia, n.71, p. 1-6.

Garcia, R. C. A (2000) Reorganização do processo de planejamento do Governo Federal: o PPA 2000-2003. Texto para Discussão N. 726, ISSN 1415-4765. Instituto de Pesquisa Econômica Aplicada - IPEA, Brasília.

Gomes, L. F. A. M., Gomes, C. F. S. e Almeida, A. T. (2002) Tomada de Decisão Gerencial: Enfoque Multicritério. ( $1^{\mathrm{a}}$ Ed.). Editora Atlas, São Paulo, SP.

Jardim, S. B. (1999) Aplicabilidade de Algumas Técnicas de Análise Multiobjetivo ao Processo Decisório no Ambito de Comitês de Gerenciamento de Bacia Hidrográfica. Dissertação do Programa de Engenharia de Recursos Hídricos e Saneamento Ambiental, Universidade Federal do Rio Grande do Sul, Porto Alegre.

Kuwahara, N. (2008) Planejamento Integrado do Setor de Transporte de Carga na Amazônia: Metodologia de Análise e Hierarquização de Alternativas de Investimentos em Infra-Estrutura de Transportes. Tese de Doutorado do Programa de Engenharia de Transpor- 
tes, Universidade Federal do Rio de Janeiro - UFRJ, Rio de Janeiro.

Kuwahara, N., Machado, W. V. e Santos, M. P. S. (2008) Tomada de Decisão em Investimentos de Infra-Estruturas de Transportes: Estudo de Caso para o Pólo Industrial de Manaus. Artigo apresentado no XXII Congresso de Pesquisa e Ensino em Transportes, ANPET, Fortaleza.

Medaglia, A. L., Hueth, D., Mendieta, J. C. e Sefair, J. A. (2008) A Multiobjective Model for the Selection and Timing of Public Enterprise Projects. Socio-Economic Planning Sciences, v. 42, n. 1, p. 31-45.

Portugal, L. S. (2006) O Conhecimento e o Planejamento dos Transportes para uma Amazônia Sustentável. In: Estudos de Transporte e Logística na Amazônia. Editora Novo Tempo, Manaus, p. 09-32.

Saaty, T. L. (1991) Método de Análise Hierárquica. Tradução e Revisão Técnica Wainer da Silveira e Silva. Editora McGraw - Hill, São Paulo, SP.

Souza, M. (1994) Breve história da Amazônia. Editora Marco Zero, São Paulo. 